\title{
Adaptive four-level modeling of laser cooling of solids
}

\author{
Weiliang Jin, ${ }^{1}$ Cheng Guo, ${ }^{2}$ Meir Orenstein, ${ }^{3}$ and Shanhui Fan ${ }^{1}$ \\ ${ }^{1)}$ Department of Electrical Engineering, Ginzton Laboratory, Stanford University, Stanford, California 94305 , \\ USA \\ ${ }^{2)}$ Department of Applied Physics, Ginzton Laboratory, Stanford University, Stanford, California 94305, \\ USA \\ 3) Department of Electrical and Computer Engineering, Technion-Israel Institute of Technology, 32000 Haifa, \\ Israel
}

(*Electronic mail: shanhui@stanford.edu)

(Dated: 8 September 2021)

Laser cooling of rare-earth doped solids has been demonstrated across a wide range of material platforms, inspiring the development of simple phenomenological models such as the four-level model to elucidate the universal properties of laser cooling under various operating conditions. However, these models usually require the input of full absorption spectra that must be provided experimentally or by additional complicated atomic modeling. In this letter, we propose that a four-level model, when extended to admit effective energy levels adaptive to the pumping photon energy, can accurately predict the cooling efficiency as a function of temperature and pumping frequency using only few inputs such as the absorption coefficient measured at a single frequency and temperature. Our model exploits the quasiequilibrium properties of the excitation of rare-earth ions for the determination of the effective four energy levels. The model is validated against published experimental results for a span of materials including ytterbium/thulium-doped glass and crystals. With the verified model, we derive explicit expressions for the optimal frequency and the operating bandwidth of pumping laser. Our model significantly simplifies the modeling process of laser cooling, and is expected to stimulate further development of optical refrigeration.

Laser cooling of solids realizes refrigeration by using antiStokes fluorescence with a fluorescence frequency higher than the pumping frequency $1-\underline{\underline{5}}$. The frequency difference corresponds to the phonon energy extracted from the solids. Compared to traditional mechanical refrigeration, laser cooling does not involve moving parts or cryogenic fluids, making it advantageous for vibration-free applications and compact cryocoolers. Intense research in recent decades has enabled laser cooling in rare-earth doped fluoride crystals to achieve a remarkable low temperature ${ }^{6}$ of $91 \mathrm{~K}$, making optical refrigeration by far the only all-solid-state cryogenic refrigeration technology.

The laser cooling process for a typical rare-earth ion involves radiative transitions of near-unity quantum yield between the ground and the excited manifolds. As illustrated in Fig.1(a), each manifold contains multiple energy levels, thus a comprehensive theory needs to capture all participating energy levels ${ }^{4,7-9}$ as well as their interactions with the phonons of the host medium ${ }^{10,11}$. To elucidate the main physics and the impact of various material and operating parameters, several simplified models such as quasi two-level models 8,12 and fourlevel models 1,13 have been developed. However, these models need extensive input of experimental data such as the detailed fluorescence/absorption spectrum of the rare-earth ions. It is therefore difficult to use these models to reveal some of the general properties of laser cooling, such as the general behaviors of many key figure of merits (FOM's). For instance, a crucial FOM is the cooling efficiency $\eta_{c}$, defined as the net cooling power normalized by the total pumping power. $\eta_{c}$ usually exhibits complicated dependence on many parameters such as pumping frequency and operating temperature. In most laser cooling systems, the optimal achievable cooling efficiency is usually assumed to be ${ }^{13,14} \eta_{c} \sim k_{B} T / \hbar \omega_{p}$ where $\omega_{p}$ is the pumping angular frequency, $T$ the operating tem- perature, and $k_{B}$ the Boltzmann constant. However, beyond a few qualitative arguments, we are not aware of any quantitative formulation to support such a basic result. As an another example, while it is known that laser cooling only occurs within a narrow range of pumping frequencies and that this bandwidth is temperature-dependent as observed experimentally ${ }^{1,13}$, a quantitative description of temperature-dependence of such bandwidth and potentially other factors that influence this bandwidth remain unknown.

The lack of a simple semi-analytical model might be attributed to the complex absorption spectra of rare-earth ions that contain many resonance features at the cryogenic temperature $(<120 \mathrm{~K})$, which was the main pursued application of laser cooling 1,4 . On the other hand, at higher temperatures, as illustrated in Fig. 1. (b), the absorption spectrum in the cooling regime (red) tends to follow a smooth exponential decay features, known as the cooling tail or the long-wavelength tail ${ }^{13}$. A simple laser cooling model developed in this domain can be beneficial for applications at room-temperature or above ${ }^{15}$, such as cooling of light-driven spacecraft ${ }^{16,17}$ and high-power lasers where heat needs to be dissipated efficiently ${ }^{8,18}-20$.

In this letter, we develop a simple semi-analytical model to capture the main features of laser cooling operating above the cryogenic temperature. Our model, termed as the adaptive four-level model, exploits the quasi-equilibrium properties of the excitation of rare-earth ions ${ }^{21}$, and extends the four-level models ${ }^{1,13}$ to admit effective energy levels that are determined by pumping photon energy. Such a phenomenological model can accurately predict the cooling efficiency over a wide range of temperatures and pumping frequencies using only few inputs including the external quantum yield, mean fluorescence frequency, parasitic absorption coefficient, and absorption coefficient of the rare-earth ions measured at a single value of temperature and frequency. The absorption spectrum in the 


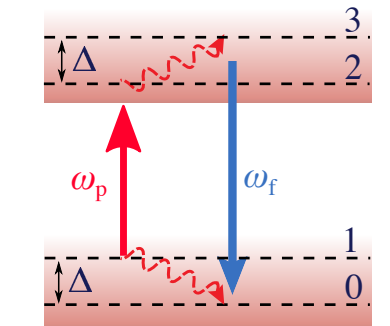

(a) Rare earth ions

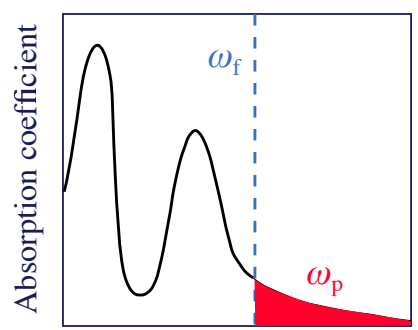

(b)

Wavelength
FIG. 1. Schematic of anti-Stokes emission from the rare-earth ions whose mean fluorescence angular frequency $\omega_{f}$ is higher than the incident laser angular frequency $\omega_{p}$. (a) The participating energy manifolds (red shaded region) are approximated here by adaptive four energy levels (black dashed line). $\omega_{i j} \equiv \omega_{i}-\omega_{j}$ denotes the angular frequency separation between Level- $i$ and Level- $j$. The model assumes $\omega_{32}=\omega_{10} \equiv \Delta$. (b) A representative absorption spectrum illustrating that the pumping frequency $\omega_{21}=\omega_{p}$, lower than $\omega_{f}$ (blue dashed line) typically occurs in the absorption tail (red region).

long-wavelength excitation regime evaluated from our model resembles the Urbach tail 22,23 . We validate our model against published experimental results in terms of absorption coefficient and cooling efficiency over a wide range of material choices including ytterbium-doped ZBLANP glass ${ }^{24,25}$, silica glass $^{12}$, YLF crystal ${ }^{26,27}$, and thulium doped ZBLANP glass $^{28}$. With our model, we prove that when maximizing the cooling power density in the ideal case, the optimal pumping angular frequency $\omega_{p, \text { opt }}$ needs to be redshifted from the mean fluorescence angular frequency $\omega_{f}$ by $\omega_{f}-\omega_{p, \text { opt }} \sim 0.8 k_{B} T / \hbar$, corresponding to a cooling efficiency $\eta_{c} \sim 0.8 k_{B} T / \hbar \omega_{p}$. Additionally, we show that the operating bandwidth, when normalized by the pumping frequency, is approximately $\sim 1.2 k_{B} T / \hbar \omega_{p}$.

We begin by reviewing the prior four-level model of rareearth doped media in the context of laser cooling 1,13 . The participating energy levels in the cooling process, as illustrated in Fig. 1 (a), lie in the two narrow manifolds called the excited and the ground manifolds, each of which contains several sublevels. The four-level model 1,13 approximates each manifold as two energy levels. Under an incident laser beam of angular frequency $\omega_{p}=\omega_{21}$ where $\omega_{i j}=\omega_{i}-\omega_{j}$ denotes the angular frequency separation between Level- $i$ and Level- $j$, electrons are excited from the upper Level-1 of the ground manifold to the lower Level-2 of the excited manifold, and then rapidly thermalized to the upper Level-3 within the excited manifold, resulting in spontaneous emission of photons of higher frequencies.

The cooling performance can be estimated from the steadystate solution of the electron occupation distribution over the four energy levels. Typically, this is done by applying the standard rate equation approach ${ }^{1,29}$. In what follows, we make the typical simplification by assuming equal inter-manifold transition rate, denoted as $\gamma / 2$, so that the lifetime of the Level$2(3)$ is $\gamma^{-1} . \quad \gamma$ is orders of magnitude lower than the intramanifold transition rate. Additionally, we assume equal width of the two manifolds, $\omega_{32}=\omega_{10}$, denoted as $\Delta$. Considering a pumping intensity $I$, the absorbed power $P_{\text {abs }}=\left[\alpha_{b}+\alpha\right] I$ is at- tributed to the parasitic (background) absorption $\alpha_{b}$ and rareearth ion absorption $\alpha$. Two important FOMs, the net cooling power density $P_{\text {cool }}$ and the cooling efficiency $\eta_{c}=\frac{P_{\text {cool }}}{P_{\text {abs }}}$, are given by (The details of the derivation can be found in Appendix $\mathrm{A}$,

$$
\begin{aligned}
& P_{\text {cool }}=\left[\eta_{e} \frac{\omega_{f}}{\omega_{p}}-1\right] \alpha I-\alpha_{b} I \\
& \eta_{c}=\eta_{e} \eta_{\mathrm{abs}} \frac{\omega_{f}}{\omega_{p}}-1
\end{aligned}
$$

Here, $\omega_{f}$ is the mean fluorescence angular frequency, $\eta_{e}=$ $\gamma_{r} / \gamma$ the external quantum yield defined as the ratio between the radiative transition rate $\gamma_{r}$ and the total transition rate $\gamma$, and $\eta_{\text {abs }}=\frac{\alpha}{\alpha+\alpha_{b}}$ the absorption efficiency. Eqs. (1-2) are wellknown, and they can also be obtained with other approaches such as the quasi two-level model ${ }^{8,12}$. Among several parameters in Eqs. (1-2), the absorption coefficient $\alpha$ is known to exhibit strong dependence on both $\omega_{p}$ and temperature. Thus, the magnitude of the two FOMs are largely dictated by $\alpha\left(\omega_{p}, T\right)$. As detailed in Appendix $\mathrm{A}$, the four-level model provides a solution for $\omega_{f}$ and $\alpha$,

$$
\begin{aligned}
& \omega_{f}=\omega_{p}+\Delta\left[\frac{1}{2}+\frac{1}{1+\exp \left[\hbar \Delta / k_{B} T\right]}\right] \\
& \alpha=\frac{\sigma_{12} N_{t}}{1+\exp \left[\hbar \Delta / k_{B} T\right]} \frac{1}{1+I / I_{s}}
\end{aligned}
$$

where $\sigma_{12}$ is the atomic absorption cross section for the transition $1 \rightarrow 2, N_{t}$ the doping concentration, and $I_{s}=\hbar \omega \gamma / \sigma_{12}$ the saturation intensity. The exponential factor $\exp \left[\hbar \Delta / k_{B} T\right]$ is attributed to the Boltzmann distribution of the electron occupation density ${ }^{29}$ across distinguishable localized rare-earth ions. Results in Eqs. (3-4) cannot be obtained with the quasi two-level model. They provide physical insights 1,13 into the temperature-dependence of the cooling performance.

However, the application of the former four-level model is limited in practice. It assumes that the manifold width $\Delta$ of the four-level system is specified by the manifold width of the actual ion, which has no relation to the incident laser frequency $\omega_{p}$. In this case, Eq. (4) does not reveal useful spectroscopic information of $\alpha$ as a function of $\omega_{p}$. Consequently, to capture $\eta_{c}$ over a range of $\omega_{p}$, the experimentally measured absorption spectrum $\alpha\left(\omega_{p}\right)$ needs to be employed in place of Eq. (4). Another modification of the four-level model is to use a phenomenological $\alpha\left(\omega_{p}\right)$ by incorporating the linewidth broadening effect ${ }^{1,13}$ into the atomic absorption cross section $\sigma_{12}$. But the determination of the linewidth function, which is the superposition of many Lorentzian and Gaussian lineshape functions ${ }^{30}$, again undermines the simplicity of the results as derived from the four-level model.

We address these limitations through better approximating the electron occupation distribution by assigning effective participating energy levels whose energy values are solved for at each $\omega_{p}$, so that the energy levels will adapt to $\omega_{p}$. The levels are determined by exploiting the quasi-equilibrium property of the electrons within each manifold of the rare-earth ions ${ }^{21}$, which will result in a $\omega_{p}$-independent mean fluorescence frequency $\omega_{f}$. This is also known as Vavilov's law based on 
empirical observations ${ }^{21,31}$. Once $\omega_{f}$ is provided, for each value of $\omega_{p}$, Eq. (3) is employed to solve for $\Delta\left(\omega_{p}\right)$, which together with $\omega_{21}=\omega_{p}$ fully determines the relative positions of the four levels. Thus, we call this model "adaptive four-level model". For a laser cooling scheme, the solution $\Delta(\omega)$ is subsequently used to obtain the spectrum $\alpha\left(\omega_{p}\right)$ via Eq. (4), and finally the cooling FOMs Eqs. (1-2). As for the number of the input parameters, in the unsaturated regime where $I \ll I_{s}$, only three $\left(\sigma_{12}, N_{t}\right.$ and $\left.\omega_{f}\right)$ are needed to determine $\alpha\left(\omega_{p}\right)$, and two more $\left(\eta_{e}\right.$ and $\left.\alpha_{b}\right)$ for $\eta_{c}$. In practice, all these five parameters can be assumed to be $\omega_{p}$-independent. The assumption of an undispersive $\sigma_{12}$ is in particular applicable to high temperature (e.g. above the cryogenic temperature) and disordered solids such as glass. The five parameters are also roughly temperature-insensitive except for $\omega_{f}$ that can exhibit a weak linear temperature dependence $\frac{1,27}{}$. Therefore, compared to the former four-level model that requires the input of $\alpha\left(\omega_{p}, T\right)$ over all relevant pumping frequencies and temperatures, our model can present great simplification, as well as more transparent description of the temperature- and $\omega_{p^{-}}$ dependence of the cooling performance.

To gain insights, we further study the model in the high phonon energy extraction region $\hbar \Delta \gg k_{B} T$ where Eqs. (3-4) can be simplified. The manifold width is $\Delta \approx 2\left(\omega_{f}-\omega_{p}\right)$, and the absorption exhibits the expected exponential decay feature,

$$
\alpha\left(\omega_{p}\right) \approx \frac{\sigma_{12} N_{t}}{1+I / I_{s}} \exp \left[-2 \hbar\left(\omega_{f}-\omega_{p}\right) / k_{B} T\right]
$$

Below we make a few observations. First, the solution $\Delta$ can exceed the actual manifold width of the rare-earth ion, indicating that Level-1 and Level-2 can lie in the phononbroadened sidebands. Second, the exponential decay feature in Eq. (5) resembles the Urbach tail in insulators and semiconductors ${ }^{22,23}$ arising from the electron-phonon interactions driven by thermal fluctuations. The absorption coefficient in the Urbach tail is proportional to $\exp \left[-\sigma \hbar\left(\Omega-\omega_{p}\right) / k_{B} T\right]$ where $\hbar \Omega$ is the effective energy gap, and $\sigma$ a constant of the order of one. Our phenomenological model corresponds to a Urbach tail of $\Omega=\omega_{f}$ and $\sigma=2$. Such a similarity can be attributed to the thermalization process present in both cases. In fact, the modeling of the optical transitions in semiconductors for photoluminescence (PL) applications ${ }^{32.33}$ is also similar to our model except for modifications such as the use of chemical potential in place of rate equations, and Fermi distribution in place of Boltzmann distribution.

We validate our model against experimental results in terms of absorption spectrum and cooling efficiency. Fig. 22 depicts the room-temperature absorption spectrum evaluated from the adaptive four-level model (red solid line), the approximated model in the high phonon energy extraction region (blue dashdotted line), and experimental results (black dashed line). For the sake of generality, we cover two main types of the host media, glass and crystals, represented by ZBLANP glass in Fig. 2 (a), and YLF crystal in Fig. 2(b), respectively. The rare-earth ion is taken as $\mathrm{Yb}^{3+}$ due to its dominance in the literature and hence the accessibility of published experimental data. The computation of the spectrum with our model involves only
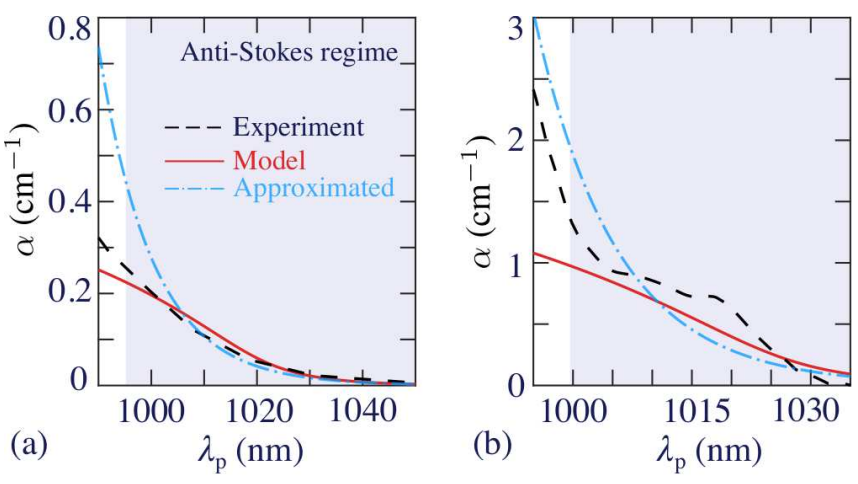

FIG. 2. The absorption spectrum at room temperature evaluated from the adaptive four-level model (red solid line), the approximated model described by Eq. [5] (blue dash-dotted line), in comparison to the experimental results (black dashed line) of (a) $\mathrm{Yb}^{3+}$ doped ZBLANP glass 25 with doping concentration $1.128 \times 10^{20} \mathrm{~cm}^{-3}$ and (b) $\mathrm{Yb}^{3+}$ doped YLF crystal 27 with doping concentration $6.98 \times$ $10^{20} \mathrm{~cm}^{-3}$. The only fitting parameter in the model is $\sigma_{12}$. The three agree well in the anti-Stokes emission regime (shaded region), where the pumping wavelength is redshifted from the mean fluorescence wavelength.

one fitting parameter $\sigma_{12}$, while the other two parameters $\omega_{f}$ and $N_{t}$ are obtained from the experimental data. The detailed values are listed in the Appendix B

Fig. 2 (a) shows that for $\mathrm{Yb}^{3+}$ doped ZBLANP glass, the measured absorption spectrum (black dashed line) indeed exhibits a smooth exponential decay lineshape in the anti-Stokes emission regime where the pumping wavelength $\lambda_{p}$ is redshifted from the mean fluorescence wavelength $\lambda_{f}$. The spectrum computed from the adaptive four-level model agrees well with the experiment in almost the entire anti-Stokes emission regime. The approximate model of Eq. (5) also exhibits good agreement when the photon energy of the incidence is redshifted from that of the fluorescence by at least one $k_{B} T$. As for $\mathrm{Yb}^{3+}$ doped YLF crystal, as shown in Fig. 2(b), the measured absorption spectrum in the anti-Stokes emission regime exhibits additional features on top of the exponential decay tail. This is because the inhomogeneous broadening, which dominates in glass, is suppressed in a crystalline media, allowing the resonance absorption peaks to be enhanced in a crystal. However, those features occurring in the long-wavelength tail are still largely suppressed since the electron occupation density on high energy levels are low according to the Boltzmann distribution. Indeed, Fig. 2 (b) elucidates that our model can still capture the general trend of the spectrum. The impact of the missing little "bumps" in our model on the cooling efficiency will be studied below.

Next, we analyze the applicability of our model in capturing cooling efficiency $\eta_{c}$. In Fig. 3, we apply our model to a wide range of material platforms in comparison to the experimental results. In particular, among the three rare-earth ions $\left(\mathrm{Yb}^{3+}, \mathrm{Tm}^{3+}\right.$, and $\left.\mathrm{Er}^{3+}\right)$ that have so far exhibited bulk cooling ${ }^{3}$, Fig. 3. 3 covers the results for both $\mathrm{Yb}^{3+}$ and $\mathrm{Tm}^{3+}$; as for the host media, the recently explored silica glass 12 is also discussed. In evaluating $\eta_{c}$, only one parameter $\sigma_{12}$ needs 

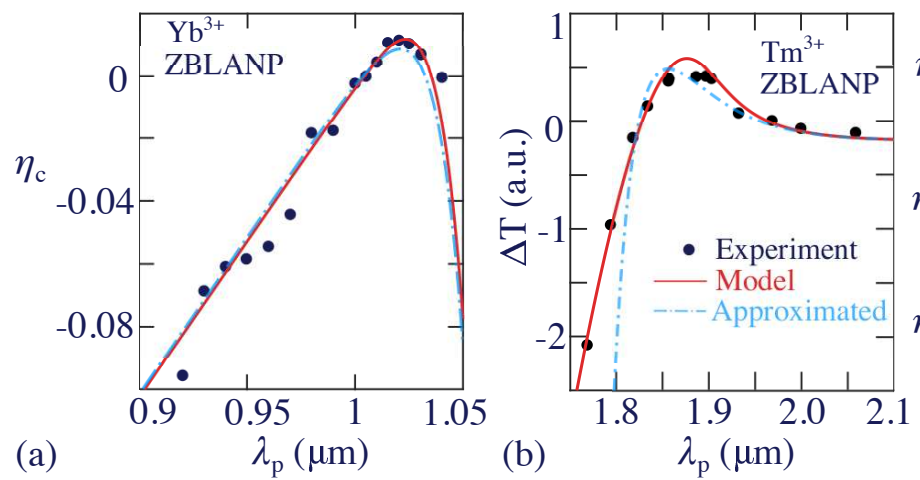

(b)

$\lambda_{\mathrm{p}}(\mu \mathrm{m})$
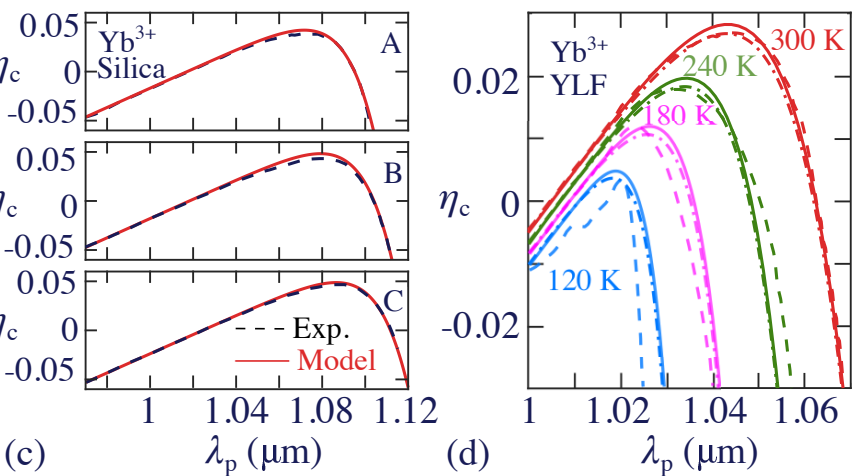

FIG. 3. The cooling efficiency $\eta_{c}$ or temperature change $\Delta T$ (proportional to $\eta_{c}$ ) as a function of pumping wavelength based on the adaptive four-level model (solid line), the approximated model (dash-dotted line), in comparison to the experimental results (black dots in (a)(b) and dashed lines in (c)(d)). Cooling occurs when $\eta_{c}>0$ or $\Delta T>0$. The material platforms are (a) $\mathrm{Yb}^{3+}$ doped ZBLANP glass ${ }^{24},(\mathrm{~b}) \mathrm{Tm}^{3+}$ doped ZBLANP glass ${ }^{28}$, (c) three samples of $\mathrm{Yb}^{3+}$ doped silica glass prepared with different fabrication techniques 12 denoted as $A, B$, and $C$, and (d) $\mathrm{Yb}^{3+}$ doped YLF crystal ${ }^{26}$ operating at various temperatures. The solid and the dashed-dotted curves in (c) coincide. The experimental results in (a)(b) are inferred directly from the temperature change measurement, while those in (c)(d) are derived from the absorption/fluorescence spectrum.
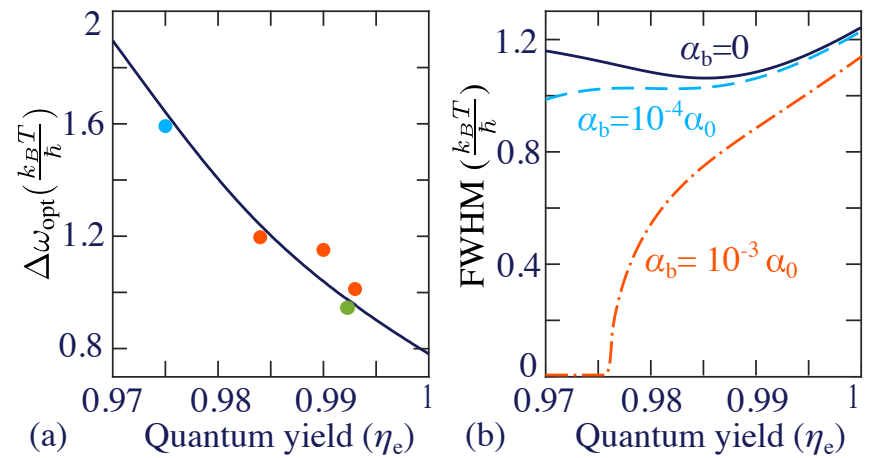

FIG. 4. (a) The optimal frequency detuning $\Delta \omega_{\text {opt }}$ of the pumping light from the mean fluorescence frequency for maximizing cooling power density as a function of external quantum yield evaluated from the adaptive four-level model (black solid line) or experimental results $12,20,24$ (dots). Dots with different colors are taken from different experimental reports, detailed in Appendix B (b) The spectral full width at half maximum of the cooling power density as a function of external quantum yield at various values of parasitic absorption rate. $\alpha_{0}$ denotes the absorption coefficient of the ion at the mean fluorescence frequency.

to be fitted ${ }^{34}$. Fig. 3 (a-c) summarize the room-temperature cooling efficiency as a function of pumping wavelength based on our model compared to experimental results for $\mathrm{Yb}^{3+}$ doped ZBLANP glass [Fig. 3 (a)], $\mathrm{Tm}^{3+}$ doped ZBLANP glass [Fig. 3 (b)], and $\mathrm{Yb}^{3+}$ doped silica glass [Fig. 3 (c)]. Remarkably, in all scenarios, the adaptive four-level model agrees well with the experiments. The approximated model performs well in Fig. 3 (a,c) involving $\mathrm{Yb}^{3+}$ ions, but deviates from the experiment at shorter wavelengths in Fig. 3 (b) related to $\mathrm{Tm}^{3+}$ ions. This is because the operating frequency of $\mathrm{Tm}^{3+}$ ions is around half compared to $\mathrm{Yb}^{3+}$ ions, making the valid regime of the high phonon energy extraction region defined in terms of $k_{B} T$ to shift to a relatively longer wavelength.
In Fig. 3(d), we further examine the accuracy of our model in revealing the temperature-dependence of $\eta_{c}$. Except for $\omega_{f}(T)$ that needs an experimental input, the impact of operating temperature in our model is explicit in Eqs. (3-4), or Eq. (5) in the long-wavelength excitation regime. Fig. 3 (d) shows that when the operating temperature is above $120 \mathrm{~K}$, the prediction of our model is very accurate. At temperature $120 \mathrm{~K}$ (blue curves) where our model is becoming less appropriate, we capture the main trend, but do not follow some fine features such as the two small peaks and slightly overestimate the cooling efficiency. As pointed out earlier, the absorption spectrum at room temperature, as shown in Fig. 2 (b), also exhibits small features that have negligible effects on the cooling efficiency. Consequently, our model is applicable also for crystalline host media.

Finally, we reveal several universal properties of laser cooling based on our adaptive four-level model. First, in a laser cooling system, it is crucial to know the optimal pumping frequency $\omega_{p, \text { opt }}$ that maximizes $P_{\text {cool }}$, and the corresponding value of $\eta_{c}$ at $\omega_{p \text {,opt }}$. Eq. (1) appears to indicate that a lower $\omega_{p}$ enhances $P_{\text {cool }}$ by increasing the extracted phonon energy. However, in practice the optimal $\hbar \omega_{p}$ is only below $\hbar \omega_{f}$ by several thermal energy $k_{B} T$ due to the diminishing of the absorption rate $\alpha\left(\omega_{p}\right)$ with frequency. In the ideal scenarios where $\eta_{e} \approx 1$ and $\alpha_{b} \approx 0$, our model shows that the peak cooling power density is achieved at an angular frequency detuning of $\Delta \omega_{p, \text { opt }} \equiv \omega_{f}-\omega_{p, \text { opt }} \approx 0.78 \frac{k_{B} T}{\hbar}$, where the cooling efficiency yields,

$$
\eta_{c} \approx 0.78 \frac{k_{B} T}{\hbar \omega_{p}}
$$

Our results provide a quantitative support to the previous claims $^{13}$ that the optimal detuning should be $\sim k_{B} T / \hbar$ and the optimal $\eta_{c} \sim k_{B} T / \hbar \omega_{p}$. As shown in Fig. 4 (a), we also validate our prediction of $\omega_{p, \text { opt }}$ (black line) under non-ideal $\eta_{e}$ against several experimental results (dots, detailed in Appendix B]. In general, at lower $\eta_{e}$, larger frequency detuning 
to increase the extracted phonon energy is needed to compensate for the heating due to the nonradiative process.

The second important quantity of laser cooling is the operating bandwidth of the pumping frequency for achieving net refrigeration, which in practice is relevant in choosing an appropriate laser. The full width at the half maximum (FWHM) of the spectral peak of $P_{\text {cool }}$ can be determined in the ideal scenario of our model as,

$$
\frac{\mathrm{FWHM}}{\omega_{p}} \approx 1.24 \frac{k_{B} T}{\hbar \omega_{p}}
$$

Eq. (7) reveals that there is a direct connection between the optimal $\eta_{c}$ described by Eq. (6) and the relative operating bandwidth of the pumping frequency. Therefore, previous efforts in enhancing $\eta_{c}$ such as operating at higher temperature 15 or exploring rare-earth ions of lower transition frequencies (e.g. compared to $\mathrm{Yb}^{3+}$ that operates at $\sim 1 \mu \mathrm{m}, \mathrm{Tm}^{3+}$ is at $\sim 2 \mu \mathrm{m}$ ) can also be applied to increase the operating bandwidth. The operating bandwidth in the non-ideal scenarios is depicted in Fig. (4 (b), showing that while the FWHM is in general on the order $k_{B} T / \hbar$, it can vanish rapidly with degrading $\eta_{e}$ when the parasitic absorption is significant.

To conclude, we have proposed a simple phenomenological model of very few inputs for capturing the spectral and thermal properties of laser cooling. Our model is validated against experimental results across several representative material platforms. Our model is mostly accurate in the presence of substantial homogeneous or inhomogeneous broadening effects where the lineshape of the absorption spectrum is mostly determined by the electron thermalization process. A potential simple model beyond this region (e.g. crystalline host media of small inhomogeneous broadening held at the cryogenic temperature for suppressing homogeneous broadening) is expected in the future.

\section{ACKNOWLEDGMENTS}

We would like to thank Dr. Wei Li and Dr. Alex Song for useful discussions. This work is supported by the Breakthrough Starshot Initiative (initial idea and performance testing), and the U.S. Department of Energy "Photonics at Thermodynamic Limits" Energy Frontier Research Center under Grant No. DE-SC0019140 (theoretical modeling).

\section{DATA AVAILABILITY STATEMENT}

The data that support the findings of this study are available from the corresponding author upon reasonable request.

\section{Appendix A: Four-level model}

Below we briefly review the rate equation formulation of laser cooling based on four-level ions $\stackrel{1}{-}$. As schematically illustrated in Fig. 1 1 of the main text, each of the manifolds of
TABLE I. Values of the parameters used to evaluate the absorption coefficient in Fig. 2 of the main text.

\begin{tabular}{lccc}
\hline \hline & $\lambda_{f}(\mathrm{~nm})$ & $N_{t}\left(10^{20} \mathrm{~cm}^{-3}\right)$ & $\sigma_{12}\left(10^{-20} \mathrm{~cm}^{-2}\right)$ \\
\hline ZBLANP glass $^{25}$ & 995 (Ref. 25) & 1.128 (Ref. 25) & 0.4 (fitted) \\
YLF crystal $^{27}$ & 999.6 (Ref. 27) & 6.98 (Ref. 27) & 0.28 (fitted) \\
\hline \hline
\end{tabular}

the ground state and the excited state is approximated as two closely-spaced energy levels. As a simple model, we assume equal width $\Delta$ for both the ground and the excited manifolds. We also assume equal degeneracy of all levels, and equal decay rate for transitions between all the excited and the ground states, namely, $\gamma_{30}=\gamma_{31}=\gamma_{21}=\gamma_{20} \equiv \frac{\gamma}{2}$. In the presence of laser pumping of frequency $\omega_{p}=\omega_{21}$ between Level-1 and 2, the electron occupation density of each energy level evolves according to the following rate equation,

$$
\begin{aligned}
& \frac{\mathrm{d} N_{0}}{\mathrm{~d} t}=\frac{\gamma}{2}\left(N_{2}+N_{3}\right)+\gamma_{10}\left(N_{1}-N_{0} \exp \left[-\hbar \Delta / k_{B} T\right]\right) \\
& \frac{\mathrm{d} N_{2}}{\mathrm{~d} t}=\mathscr{P}\left(N_{1}-N_{2}\right)-\gamma N_{2}+\gamma_{32}\left(N_{3}-N_{2} \exp \left[-\hbar \Delta / k_{B} T\right]\right) \\
& \frac{\mathrm{d} N_{3}}{\mathrm{~d} t}=-\gamma N_{3}-\gamma_{32}\left(N_{3}-N_{2} \exp \left[-\hbar \Delta / k_{B} T\right]\right) \\
& N_{0}+N_{1}+N_{2}+N_{3}=N_{t}
\end{aligned}
$$

where $N_{t}$ is the total electron density, and $\mathscr{P}=\frac{I \sigma_{12}}{\hbar \omega_{p}}$ is the pumping rate that depends on the laser intensity $I$ and ion absorption cross section $\sigma_{12}$ of the Level $1 \rightarrow 2$ transition. For rare-earth ions, we apply the approximate $\gamma \ll \gamma_{32}\left(\gamma_{10}\right)$ since the lifetime $1 / \gamma$ is typically on the order of $10^{-6} \sim 10^{-3} \mathrm{~s}$, while $1 / \gamma_{32}\left(\gamma_{10}\right)$ is around $10^{-12} \sim 10^{-9} \mathrm{~s}$, as seen in Ref. $\underline{3}$. The stationary solution relevant to laser cooling yields,

$$
\begin{aligned}
& N_{1}-N_{2}=\frac{N_{t}}{\left(1+\frac{\mathscr{P}}{\gamma}\right)\left(1+\exp \left[\hbar \Delta / k_{B} T\right]\right)} \\
& N_{2}+N_{3}=\frac{\mathscr{P}}{\gamma}\left(N_{1}-N_{2}\right) \\
& N_{2}=\frac{\mathscr{P}}{\gamma} \frac{N_{1}-N_{2}}{\left(1+\exp \left[-\hbar \Delta / k_{B} T\right]\right)}
\end{aligned}
$$

With the foregoing expressions, the absorbed power and emitted power can be expressed as,

$$
\begin{aligned}
& P_{\mathrm{abs}}=\mathscr{P} \hbar \omega\left(N_{1}-N_{2}\right)=\alpha I \\
& P_{\mathrm{emit}}=\left(N_{2}+N_{3}\right) \hbar \omega_{f} \gamma_{r}=\eta_{e} \frac{\omega_{f}}{\omega_{p}} \alpha I
\end{aligned}
$$

where $\gamma_{r}$ is the radiative decay rate, and the expression of $\alpha$ can be found in the main text. The mean fluorescence frequency $\omega_{f}$ in our four-level system is the average transition frequency weighted by the occupation density,

$$
\begin{aligned}
\omega_{f} & =\frac{1}{2}\left[\frac{N_{2}}{N_{2}+N_{3}}\left(\omega_{21}+\omega_{20}\right)+\frac{N_{3}}{N_{2}+N_{3}}\left(\omega_{31}+\omega_{30}\right)\right] \\
& =\omega_{p}+\Delta\left[\frac{1}{2}+\frac{1}{1+\exp \left[\hbar \Delta / k_{B} T\right]}\right]
\end{aligned}
$$


TABLE II. Values of the parameters used to evaluate the cooling efficiency in Fig. 3 of the main text.

\begin{tabular}{|c|c|c|c|c|c|}
\hline & $\lambda_{f}(\mathrm{~nm})$ & $N_{t}\left(10^{20} \mathrm{~cm}^{-3}\right)$ & $\eta_{e}$ & $\alpha_{b}\left(10^{-4} \mathrm{~cm}^{-1}\right)$ & $\sigma_{12}\left(10^{-20} \mathrm{~cm}^{-2}\right)$ \\
\hline $\mathrm{Yb}^{3+}-\mathrm{ZBLANP}$ glass $^{24}$ & 995 (Ref. 24) & 2.42 (Ref. 24) & 0.992 (fitted) & 0.8 (fitted) & 0.037 (fitted) \\
\hline $\mathrm{Yb}^{3+}-\mathrm{YLF}$ crystal 26 & 1008.9-0.031T (Ref. 27) & 6.98 (Ref. 26) & 0.995 (Ref. 26) & 4 (Ref. 26) & 0.28 (fitted) \\
\hline $\mathrm{Yb}^{3+}-$ silica glass $\mathrm{A}^{12}$ & 1010 (Ref. 12) & 0.53 (Ref. 12) & 0.993 (Ref. 12) & 0.23 (Ref. 12) & 0.9 (fitted) \\
\hline $\mathrm{Yb}^{3+}-$ silica glass $\mathrm{B}^{12}$ & 1008 (Ref. 12) & 0.44 (Ref. 12) & 0.990 (Ref. 12) & 0.21 (Ref. 12) & 2.2 (fitted) \\
\hline $\mathrm{Yb}^{3+}$ - silica glass $\mathrm{C}^{12}$ & 1008 (Ref. 12) & 0.57 (Ref. 12) & 0.984 (Ref. 12) & 0.12 (Ref. 12$)$ & 1.7 (fitted) \\
\hline $\mathrm{Tm}^{3+}$ - ZBLANP glass 28 & 1820 (Ref. 28) & 2.42 (Ref. 28) & 0.9997 (fitted) & 2 (fitted) & 0.042 (fitted) \\
\hline
\end{tabular}

TABLE III. Values of the parameters used to evaluate the data points of the optimal pumping frequency detuning $\Delta \omega_{\text {opt }}=\omega_{f}-\omega_{p, \text { opt }}$ as a function of $\eta_{e}$ in Fig. 4 (a) of the main text, where $\omega_{p, \text { opt }}$ is the pumping frequency that optimizes the cooling power.

\begin{tabular}{lccc}
\hline \hline & $\eta_{e}$ & $\lambda_{f}(\mathrm{~nm})$ & $\lambda_{p, \text { opt }}(\mathrm{nm})$ \\
\hline $\mathrm{Yb}^{3+}$ doped silica glass $\mathrm{A}^{12}$ (red dot) & 0.993 (Ref. 12) & 1010 (Ref. 12) & 1032 (Read from the figure in Ref. 12) \\
$\mathrm{Yb}^{3+}$ doped silica glass B 12 (red dot) & 0.990 (Ref. 12) & 1008 (Ref. 12) & 1033 (Read from the figure in Ref. 12) \\
$\mathrm{Yb}^{3+}$ doped silica glass C12 (red dot) & 0.984 (Ref. 12) & 1008 (Ref. 12) & 1034 (Read from the figure in Ref. 12) \\
$\mathrm{Yb}^{3+}$ doped silica glass & 10 \\
(blue dot) & 0.975 (Ref. 20) & 1002.5 (Ref. 20) & 1037 (Ref. 20) \\
$\mathrm{Yb}^{3+}$ doped ZBLANP glass 24 (green dot) & 0.992 (fitted) & 995 (Ref. 24) & 1015 (Ref. 24) \\
\hline \hline
\end{tabular}

\section{Appendix B: Parameter values}

Table (I-III) list parameter values for Fig.2, Fig.3, and Fig.4 (a), respectively.

${ }^{1}$ D. V. Seletskiy, M. P. Hehlen, R. I. Epstein, and M. Sheik-Bahae, "Cryogenic optical refrigeration," Advances in Optics and Photonics 4, 78-107 (2012).

${ }^{2}$ G. Nemova and R. Kashyap, "Twenty years of laser cooling of solids," in Journal of Physics: conference series, Vol. 619 (Institute of Physics and IOP Publishing Limited, 2015) p. 012037.

${ }^{3}$ M. P. Hehlen, M. Sheik-Bahae, and R. I. Epstein, "Solid-state optical refrigeration," (Elsevier, 2014).

${ }^{4}$ G. Nemova, Laser Cooling: Fundamental Properties and Applications (CRC Press, 2016).

${ }^{5}$ D. Ding, T. Kim, and A. Minnich, "Active thermal extraction of near-field thermal radiation," Physical Review B 93, 081402 (2016).

${ }^{6}$ S. D. Melgaard, A. R. Albrecht, M. P. Hehlen, and M. Sheik-Bahae, "Solidstate optical refrigeration to sub-100 kelvin regime," Scientific Reports 6, 20380 (2016).

${ }^{7}$ G. Lamouche, P. Lavallard, R. Suris, and R. Grousson, "Low temperature laser cooling with a rare-earth doped glass," Journal of Applied Physics 84, 509-516 (1998).

${ }^{8}$ J. M. Knall, M. Esmaeelpour, and M. J. Digonnet, "Model of anti-stokes fluorescence cooling in a single-mode optical fiber," Journal of Lightwave Technology 36, 4752-4760 (2018).

${ }^{9}$ G. Nemova and R. Kashyap, "Optimization of optical refrigaration in yb3+:yag samples," Journal of Luminescence 164, 99 - 104 (2015).

${ }^{10}$ J. Fernández, A. Mendioroz, A. García, R. Balda, J. Adam, and M. Arriandiaga, "On the origin of anti-stokes laser-induced cooling of $\mathrm{Yb}^{3+}$-doped glass," Optical Materials 16, 173-179 (2001).

${ }^{11} \mathrm{~J}$. Kim, A. Kapoor, and M. Kaviany, "Material metrics for laser cooling of solids," Physical Review B 77, 115127 (2008).

${ }^{12}$ E. Mobini, S. Rostami, M. Peysokhan, A. Albrecht, S. Kuhn, S. Hein, C. Hupel, J. Nold, N. Haarlammert, T. Schreiber, et al., "Laser cooling of ytterbium-doped silica glass," Communications Physics 3, 134 (2020).

${ }^{13}$ D. V. Seletskiy, R. Epstein, and M. Sheik-Bahae, "Laser cooling in solids: advances and prospects," Reports on Progress in Physics 79, 096401 (2016).

${ }^{14}$ M. Sheik-Bahae and R. I. Epstein, "Laser cooling of solids," Laser \& Photonics Reviews 3, 67-84 (2009).

${ }^{15}$ Y. Nakayama, Y. Harada, and T. Kita, "Improving laser cooling efficiencies of yb-doped yttrium aluminum garnet by utilizing non-resonant anti-stokes emission at high temperatures," Optics Express 27, 34961-34973 (2019).
${ }^{16}$ H. A. Atwater, A. R. Davoyan, O. Ilic, D. Jariwala, M. C. Sherrott, C. M. Went, W. S. Whitney, and J. Wong, "Materials challenges for the starshot lightsail," Nature Materials 17, 861 (2018).

${ }^{17} \mathrm{~W}$. Jin and et al, To be published.

${ }^{18}$ S. R. Bowman, "Lasers without internal heat generation," Journal of Quantum Electronics 35, 115-122 (1999).

${ }^{19}$ E. Balliu, A. Thontakudi, J. M. Knall, and M. J. Digonnet, "Predictive comparison of anti-stokes fluorescence cooling in oxide and non-oxide fiber hosts doped with $\mathrm{Er}^{3+}$ or $\mathrm{Yb}^{3+}$, , in Photonic Heat Engines: Science and Applications, Vol. 10936 (International Society for Optics and Photonics, 2019) p. 109360J.

${ }^{20}$ J. Knall, P.-B. Vigneron, M. Engholm, P. D. Dragic, N. Yu, J. Ballato, M. Bernier, and M. J. Digonnet, "Laser cooling in a silica optical fiber at atmospheric pressure," Optics Letters 45, 1092-1095 (2020).

${ }^{21}$ M. J. Digonnet, Rare-earth-doped fiber lasers and amplifiers, revised and expanded (CRC press, 2001).

${ }^{22} \mathrm{~F}$. Urbach, "The long-wavelength edge of photographic sensitivity and of the electronic absorption of solids," Physical Review 92, 1324 (1953).

${ }^{23}$ Y. Toyozawa, "A proposed model for the explanation of the urbach rule," Progress of Theoretical Physics 22, 455-457 (1959).

${ }^{24}$ C. Mungan, M. Buchwald, B. Edwards, R. Epstein, and T. Gosnell, "Laser cooling of a solid by $16 \mathrm{k}$ starting from room temperature," Physical Review Letters 78, 1030 (1997).

${ }^{25}$ G. Lei, J. E. Anderson, M. I. Buchwald, B. C. Edwards, R. I. Epstein, M. T. Murtagh, and G. Sigel, "Spectroscopic evaluation of $\mathrm{Yb}^{3+}$-doped glasses for optical refrigeration," IEEE Journal of Quantum Electronics 34, 1839 1845 (1998).

${ }^{26}$ D. V. Seletskiy, S. D. Melgaard, R. I. Epstein, A. Di Lieto, M. Tonelli, and M. Sheik-Bahae, "Local laser cooling of Yb: YLF to $110 \mathrm{k}$," Optics Express 19, 18229-18236 (2011).

${ }^{27}$ D. V. Seletskiy, S. D. Melgaard, R. I. Epstein, A. Di Lieto, M. Tonelli, and M. Sheik-Bahae, "Precise determination of minimum achievable temperature for solid-state optical refrigeration," Journal of Luminescence 133, 5-9 (2013).

${ }^{28}$ C. Hoyt, M. Sheik-Bahae, R. Epstein, B. Edwards, and J. Anderson, "Observation of anti-stokes fluorescence cooling in thulium-doped glass," Physical Review Letters 85, 3600 (2000).

${ }^{29}$ A. E. Siegman, Lasers (University Science Books, 1986).

${ }^{30}$ R. M. Martin, Reciprocity between emission and absorption for rare earth ions in glass (Worcester Polytechnic Institute, 2006).

${ }^{31}$ A. Rayner, N. R. Heckenberg, and H. Rubinsztein-Dunlop, "Condensedphase optical refrigeration," Journal of the Optical Society of America B 20, 1037-1053 (2003).

${ }^{32}$ P. Wurfel, "The chemical potential of radiation," Journal of Physics C: Solid State Physics 15, 3967 (1982). 
${ }^{33}$ M. Cardona and Y. Y. Peter, Fundamentals of semiconductors (Springer, 2005).
${ }^{34}$ When the values of $\alpha_{b}$ and $\eta_{e}$ are missing in the experimental reference, we obtain these values by fitting. 